\title{
On the convergence, consistence and stability of a standard finite difference scheme
}

\author{
${ }^{* 1}$ Prof. Emmanuel Adeolu Ibijola and ${ }^{2}$ Joshua Sunday \\ ${ }^{* 1}$ Corresponding Author: Department of Mathematical sciences, University of \\ Ado-Ekiti, P. M. B. 5363, Ekiti State, Nigeria (+2348035891025, \\ ibjemm@yahoo.com) \\ ${ }^{2}$ Department of Mathematical sciences, Adamawa State University, P.M. B. 25, \\ Mubi-Nigeria
}

\begin{abstract}
For any numerical method to be efficient, ingenious and computationally reliable, it is expected that it be convergent, consistent and stable. In this paper, we investigate the convergence, consistency and stability of a Standard Finite-Difference Scheme developed by Ibijola and Sunday (2010). This scheme has also been shown to be applicable in solving Ordinary Differential Equations.
\end{abstract}

Keywords: Standard Finite-Difference Scheme, Convergence, Consistency, Stability and Initial Value Problems

\section{INTRODUCTION}

It is a known and documented fact that a given linear or non-linear equation does not have a complete solution that can be expressed in terms of a finite number of elementary functions. It is also a known fact that one of the ways to solve such problem is to seek an approximate solution by means of various perturbation methods (Ross 1964, Humi and Miller 1989). It must be stated here that the above procedure will only hold for limited ranges of the system parameters and the independent variable (Mickens, 1994). As reported in Mickens(1994), for arbitrary values of the system parameters at the present time, only numerical integration technique can provide accurate solutions to the original differential equation.

It is because of the above facts that Ibijola and Sunday (2010) developed a new Standard Finite Difference Scheme, capable of solving equations of the form;

$y^{\prime}=f(x, y), y\left(x_{0}\right)=y_{0}, x \in[a, b]$

Throughout, we shall consider equations of the form (1) which occur in physical and biological sciences, management sciences and engineering. Infact, the importance of solving equations of the form (1) cannot be over emphasized. This is because of the fact that many processes in the fields mentioned above are governed by the equations of the form (1).

Let us consider theorem 1 below which guarantees the existence of a unique solution of any initial value problem of the nature (1).

\section{Theorem 1 (Lambert 1973, Fatunla 1988)}

Let $f(x, y)$ be defined and continuous for all points $(x, y)$ in the region $D$ defined by $a \leq x \leq b,-\infty<y<\infty, a$ and $b$ finite, and let there exist a constant $L$ such that for every $x, y, y^{*}$ such that $(x, y)$ and $\left(x, y^{*}\right)$ are both in $D$;

$\left|f(x, y)-f\left(x, y^{*}\right)\right| \leq L|y-y *|$

Then if $\eta$ is any given number, there exist a unique solution $y(x)$ of the initial value problem (1). The inequality (2) is known as a Lipschitz condition and the constant $L$ as a Lipschitz constant.

\section{Definition 1 (Henrici 1962)}

Any method for solving a differential equation in which the approximation $y_{n+1}$ to the solution at the point $x_{n+1}$ can be calculated if only $x_{n}, y_{n}$, and $h$ is 
known as a ONE-STEP METHOD. It is a common fact to write the functional dependence $y_{n+1}$ on the quantities $x_{n}, y_{n}$, and $h$ in the form;

$y_{n+1}=y_{n}+h \phi\left(x_{n}, y_{n} ; h\right)$

where $\phi\left(x_{n}, y_{n} ; h\right)$ is the increment function.

\section{Definition 2 (Convergence)}

A numerical scheme is said to be convergent if for all initial value problem (1) satisfying the hypothesis of Lipschitz condition,

$\underset{0 \leq n \leq N}{\operatorname{Max}}\left\|y\left(x_{n}\right)-y_{n}\right\| \rightarrow 0$ as $h \rightarrow 0$ or if for any arbitrary point $x \in[a, b]$, the global error fulfills the following relationship; $\lim _{h \rightarrow 0} \operatorname{Max}_{n} \rightarrow 0$, provided $x$ is always a mesh point.

\section{Definition 3 (Consistence)}

A numerical scheme with an increment function $\phi\left(x_{n}, y_{n} ; h\right)$ is said to be consistent with the initial value problem (1), if $\phi\left(x_{n}, y_{n} ; 0\right)=f\left(x_{n}, y_{n}\right)$. Note that if the scheme developed is consistent with the initial value problem (1), then, $h=0$. The concept of consistency of one-step method is very crucial in the sense that it controls the magnitude of the local truncation error.

\section{STABILITY (Henrici 1962)}

A numerical solution of the class of system (1) is said to be stable if the difference between the numerical solution and the theoretical solution can be made as small as possible, i.e. if there exists two positive numbers $e_{0}$ and $K$ such that the following holds;

$$
\left\|y_{n}-y\left(x_{n}\right)\right\| \leq K\left\|e_{0}\right\|
$$

\section{Theorem 2}

Let $y_{n}=y\left(x_{n}\right)$ and $l_{n}=l\left(x_{n}\right)$ denote two different solutions of our differential equation under consideration, with the initial condition specified as $y\left(x_{0}\right)=\eta$ and $l\left(x_{0}\right)=\eta^{*}$ respectively, such that $\left|l-l^{*}\right|<\varepsilon, \varepsilon>0$. If the two numerical estimates are generated by;

$$
\begin{aligned}
& y_{n+1}=y_{n}+h \phi\left(x_{n}, y_{n} ; h\right) \\
& l_{n+1}=l_{n}+h \phi\left(x_{n}, l_{n} ; h\right)
\end{aligned}
$$

The condition that;

$$
\left|y_{n+1}-l_{n+1}\right| \leq K\left|\eta-\eta^{*}\right|
$$

is the necessary and sufficient condition that a numerical scheme be stable and convergent.

We shall now proceed to consider the derivation, convergence, consistency, stability and applications of the new Standard Finite Difference Scheme.

\section{Derivation of the new standard finite-difference scheme (Ibijola and Sunday 2010)}

Let us assume that the theoretical solution $y(x)$ to the initial value problem (1) can be locally represented in the interval $\left[x_{n}, x_{n+1}\right], n \geq 0$ by the non-polynomial interpolating function;

$F(x)=a_{0}+a_{1} e^{\alpha x}, \quad$ where $\quad a_{0}, a_{1}$, and $\alpha$ are constants

At the point $x=x_{n}$,we have

$$
F\left(x_{n}\right)=a_{0}+a_{1} e^{\alpha x_{n}}
$$

While at the point $x=x_{n+1}$, equation (8) becomes,

$$
F\left(x_{n+1}\right)=a_{0}+a_{1} e^{\alpha x_{n+1}}
$$

Differentiating (8) gives;

$$
F^{\prime}(x)=a_{1} \alpha e^{\alpha x}
$$

and;

$$
F^{\prime}\left(x_{n}\right)=a_{1} \alpha e^{\alpha x_{n}}
$$

We further assume that (12) coincides with (1) and hence we have;

$$
\begin{aligned}
& a_{1} \alpha e^{\alpha x_{n}}=f\left(x_{n}, y_{n}\right) \\
& \Rightarrow a_{1}=\frac{f\left(x_{n}, y_{n}\right)}{\alpha e^{\alpha x_{n}}}
\end{aligned}
$$

To proceed further, we assume that the theoretical and numerical solution initially coincide, hence

$$
\begin{aligned}
& F\left(x_{n}\right)=y_{n} \\
& F\left(x_{n+1}\right)=y_{n+1}
\end{aligned}
$$


Using (9), (10), (14) and (15), we have

$$
\begin{aligned}
& y_{n+1}-y_{n}=a_{1}\left(e^{\alpha x_{n+1}}-e^{\alpha x_{n}}\right) \\
& \Rightarrow y_{n+1}=y_{n}+\frac{\left(e^{\alpha x_{n+1}}-e^{\alpha x_{n}}\right) f\left(x_{n}, y_{n}\right)}{\alpha e^{\alpha x_{n}}}
\end{aligned}
$$

Using the fact that $x_{n}=$ nh and $x_{n+1}=(n+1) h$, equation (17) becomes;

$$
y_{n+1}=y_{n}+\frac{f_{n}}{\alpha}\left(e^{\alpha h}-1\right)
$$

Equation (18) is the required numerical scheme. We must mention here that this numerical method is strong enough to solve problems of the form (1). Equation (18) is the new Standard Finite-Difference Scheme and it is a one-step method.

Convergence of the new standard finite difference scheme: Let us start by establishing the fact that our new numerical scheme in equation (18) can be expressed as a one-step method in the form of equation (3), where $\phi\left(x_{n}, y_{n} ; h\right)$ is the increment function. We shall now proceed to derive the increment function.

At $x=x_{n}$ and $x=x_{n+1}$, equation (8) becomes;

$$
F\left(x_{n}\right)=a_{0}+a_{1} e^{\alpha x_{n}}
$$

and

$$
F\left(x_{n+1}\right)=a_{0}+a_{1} e^{\alpha x_{n+1}}
$$

If we assume that $F\left(x_{n}\right)$ and $F\left(x_{n+1}\right)$, coincides with $y_{n}$ and $y_{n+1}$ respectively and $F^{(i)}$ denote the ith total derivatives of $f(x, y)$ with respectively to $x_{n}$, we have

$$
F^{\prime}\left(x_{n}\right)=\alpha a_{1} e^{\alpha x_{n}}=f_{n}
$$

Solving for $a_{1}$ in (21) gives

$$
a_{1}=\frac{f_{n}}{\alpha e^{\alpha x_{n}}}
$$

Considering the assumptions in equations (19) and (20) as $F\left(x_{n}\right)=y_{n}$ and $F\left(x_{n+1}\right)=y_{n+1}$ respectively we have our first numerical integrator generated as

$$
y_{n+1}=y_{n}+\frac{f_{n}}{\alpha}\left(e^{\alpha h}-1\right)
$$

Expanding $e^{\alpha h}$ in series form we have;

$$
e^{\alpha h}=\sum_{r=0}^{\infty} \frac{(\alpha h)^{r}}{(r !)}=1+\alpha h+\frac{\alpha^{2} h^{2}}{2 !}+\frac{\alpha^{3} h^{3}}{3 !}+\ldots
$$

Substituting equation (24) into (23) and expanding it will give us the following equation;

$$
\begin{gathered}
y_{n+1}=y_{n}+\frac{f_{n}}{\alpha}\left(1+\alpha h+\frac{\alpha^{2} h^{2}}{2 !}+\frac{\alpha^{3} h^{3}}{3 !}+\ldots-1\right) \\
y_{n+1}=y_{n}+f_{n}\left(h+\frac{\alpha h^{2}}{2 !}+\frac{\alpha^{2} h^{3}}{3 !}+\ldots\right) \\
y_{n+1}=y_{n}+h f_{n}\left(1+\frac{\alpha h}{2 !}+\frac{\alpha^{2} h^{2}}{3 !}+\ldots\right)
\end{gathered}
$$

Let

$$
A=1+\frac{\alpha h}{2 !}+\frac{\alpha^{2} h^{2}}{3 !}+\ldots
$$

Then our numerical scheme can be written compactly as

$$
y_{n+1}=y_{n}+h f_{n} A
$$

where

$$
\phi\left(x_{n}, y_{n} ; h\right)=f_{n} A
$$

is the increment function. We shall now proceed to show that our method (18) is convergent. This is one of the findings of this work.

\section{Theorem 3}

Let the increment function of the scheme (18) defined by (30) be the function of arguments in the region $D$ defined $x \in[a, b], y \in(-\infty, \infty), 0 \leq h \leq h_{0}$, where $h_{0}>0$ and let there exists a constant $L$, then,

$$
\left|\phi\left(x_{n}, y_{n}^{*} ; h\right)-\phi\left(x_{n}, y_{n} ; h\right)\right| \leq L\left|y_{n}^{*}-y_{n}\right|
$$


for all $\left(x_{n}, y_{n} ; h\right)$ and $\left(x_{n}, y_{n}^{*} ; h\right)$ in the region just defined. This is the necessary and sufficient condition for the convergence of the method (18).

\section{Proof}

Recall that from (30), the increment function $\phi\left(x_{n}, y_{n} ; h\right)=f_{n} A$, where

$$
A=1+\frac{\alpha h}{2 !}+\frac{\alpha^{2} h^{2}}{3 !}+\ldots
$$

Considering equation (30), we can also write

$$
\phi\left(x_{n}, y_{n} ; h\right)=A f\left(x_{n}, y_{n}\right)
$$

and

$$
\begin{gathered}
\phi\left(x_{n}, y_{n}^{*} ; h\right)=A f\left(x_{n}, y_{n}^{*}\right) \\
\phi\left(x_{n}, y_{n}^{*} ; h\right)-\phi\left(x_{n}, y_{n} ; h\right)=A\left[f\left(x_{n}, y_{n}^{*}\right)-f\left(x_{n}, y_{n}\right)\right]
\end{gathered}
$$

Let $\bar{y}$ be defined as a point in the interior of the interval whose end points are $y$ and $y^{*}$, applying Mean Value Theorem, we have

$$
f\left(x_{n}, y_{n}^{*}\right)-f\left(x_{n}, y_{n}\right)=\frac{\partial f\left(x_{n}, \bar{y}\right)}{\partial y_{n}}\left(y_{n}^{*}-y_{n}\right)
$$

If we define

$$
\begin{aligned}
& M=\operatorname{Sup}_{\left(x_{n}, y_{n}\right) \in D} \frac{\partial f\left(x_{n}, y_{n}\right)}{\partial y_{n}} \\
& \not\left(x_{n}, y_{n}^{*} ; h\right)-\phi\left(x_{n}, y_{n} ; h\right)=A \frac{\partial f\left(x_{n}, \bar{y}\right)}{\partial y_{n}}\left(y_{n}^{*}-y_{n}\right) \\
& \phi\left(x_{n}, y_{n}^{*} ; h\right)-\phi\left(x_{n}, y_{n} ; h\right)=A M\left(y_{n}^{*}-y_{n}\right)
\end{aligned}
$$

Taking the absolute values of both sides give; $\left|\phi\left(x_{n}, y_{n}^{*} ; h\right)-\phi\left(x_{n}, y_{n} ; h\right)\right| \leq|A M|\left|y_{n}^{*}-y_{n}\right|$

If we let $L=|A M|$, then equation (40) turns to

$\left|\phi\left(x_{n}, y_{n}^{*} ; h\right)-\phi\left(x_{n}, y_{n} ; h\right)\right| \leq L\left|y_{n}^{*}-y_{n}\right|$

which is the condition for convergence.

\section{Consistency of the new standard finite-difference scheme}

Recall Fatunla (1988), that says a numerical scheme with an increment function of $\phi\left(x_{n}, y_{n} ; h\right)$ is consistence with respect to the initial value problem (1) if

$$
\phi\left(x_{n}, y_{n} ; 0\right)=f\left(x_{n}, y_{n}\right)
$$

We say that our new numerical scheme is consistent since (18) reduces to (42) when $h=0$ in the equation below.

$$
\phi\left(x_{n}, y_{n} ; h\right)=f_{n}\left(1+\frac{\alpha h}{2 !}+\frac{\alpha^{2} h^{2}}{3 !}+\ldots\right)
$$

which is obtained from equation (27). Therefore, the scheme is consistent.

\section{Stability analysis of the new standard-finite difference scheme}

\section{Theorem 4}

Let $y_{n}=y\left(x_{n}\right)$ and $l_{n}=l\left(x_{n}\right)$ denote two different numerical solutions of differential equation (1) with the initial conditions specified as $y\left(x_{0}\right)=\eta$ and $l\left(x_{0}\right)=\eta *$ respectively, such that $\left|\eta-\eta^{*}\right|<\varepsilon, \varepsilon>0$. If the two numerical estimates are generated by the interpolation scheme (27), we have

$$
\begin{aligned}
& y_{n+1}=y_{n}+h \phi\left(x_{n}, y_{n} ; h\right) \\
& l_{n+1}=l_{n}+h \phi\left(x_{n}, l_{n} ; h\right)
\end{aligned}
$$

The condition that

$$
\left|y_{n+1}-l_{n+1}\right| \leq k\left|\eta-\eta^{*}\right|
$$

is the necessary and sufficient conditions that our numerical scheme (18) be stable and convergent. 


\section{Proof}

Let

$$
y_{n+1}=y_{n}+h A f\left(x_{n}, y_{n}\right)
$$

and

$$
l_{n+1}=l_{n}+h A f\left(x_{n}, l_{n}\right)
$$

then,

$y_{n+1}-l_{n+1}=y_{n}-l_{n}+h A\left[f\left(x_{n}, y_{n}\right)-f\left(x_{n}, l_{n}\right)\right]$

Let $\bar{y}$ be a point in the interior of the interval whose end points are $y$ and $l$, if we apply the Mean Value Theorem, as before, we have

$$
f\left(x_{n}, y_{n}\right)-f\left(x_{n}, l_{n}\right)=\frac{\partial f\left(x_{n}, \bar{y}\right)}{\partial y_{n}}\left(y_{n}-l_{n}\right)
$$

Let us define

$$
L=\frac{\partial f\left(x_{n}, \bar{y}\right)}{\partial y_{n}}
$$

Then,

$$
\begin{aligned}
& y_{n+1}-l_{n+1}=y_{n}-l_{n}+h A \frac{\partial f\left(x_{n}, \bar{y}\right)}{\partial y_{n}}\left(y_{n}-l_{n}\right) \\
& y_{n+1}-l_{n+1}=y_{n}-l_{n}+h A L\left(y_{n}-l_{n}\right)
\end{aligned}
$$

Taking the absolute value of both sides of equation (53) gives

$$
\left|y_{n+1}-l_{n+1}\right|=\left|\left(y_{n}-l_{n}\right)+h A L\left(y_{n}-l_{n}\right)\right| \leq|1+h A L|\left|y_{n}-l_{n}\right|
$$

Given $\quad \varepsilon>0, \quad$ and $\quad y\left(x_{n}\right)=\eta, l\left(x_{n}\right)=\eta^{*}$ and $|1+h A L|=K$, then

$$
\left|y_{n+1}-l_{n+1}\right| \leq K\left|\eta-\eta^{*}\right|
$$

Then we conclude that our method (18) is stable and hence convergent.

\section{CONCLUSION}

Since, it has been established that the scheme is convergent, consistent and stable, it is obvious its numerical solution will show a measure of convergence towards the exact (theoretical) solution, especially as the step-length tends to zero. For application of the scheme (18), refer to Ibijola, E. A. and Sunday, J. (2010), Australian Journal of Basic and Applied Science, 4(4): 624-632.

\section{REFERENCES}

Fatunla, S. O. (1976), "A New Algorithm for Numerical Solution of Ordinary Differential Equations". Computer and Mathematics with Applications, 2:247-253.

Fatunla, S. O. (1988), "Numerical Methods for Initial Value Problems in Ordinary Differential Equations". Academic Press Inc, New York.

Gakhov, F. D. (1966), "Boundary Value Problems". Dover Publications Inc, New York.

Henrici, P. (1962), "Discrete Variable Methods in Ordinary Differential Equations". John Wiley and Sons, New York.

Humi, M. and Miller, W. (1988), "Second Course in Ordinary Differential Equations for Scientists and Engineers". Springer-Verlag; New York.

Ibijola, E. A. and Sunday, J. (2010), "A Comparative Study of Standard and Exact Finite-Difference Schemes for Numerical Solution of Ordinary Differential Equations Emanating from the Radioactive Decay of Substances". Australian Journal of Basic and Applied Sciences, 4(4): 624-632.

Lambert, J. D.(1973), "Computational Methods in Ordinary Differential Equations". John Willey and Sons, New York.

Lambert, J. D. (1991), "Numerical Methods for Ordinary Differential Systems: The Initial Value Problem". John Willey and Sons, New York.

Mickens, R. E. (1981), "Non-Linear Oscillations". Cambridge University Press, New York.

Mickens, R. E. (1990), "Difference Equations; Theory and Applications". Van Nonstrand Reinhold, New York.

Mickens, R. E. (1994), "Non-Standard Finite Difference Models of Differential Equations". World Scientific, Singapore.

Mickens, R. E. (1999), "Applications of Non-Standard Method for Initial Value Problems". World Scientific, Singapore.

Ross, S. L. (1964), "Difference Equations". Blaisdeu; Waltham, MA. 